\section{DRUG THERAPY IN PYELITIS *}

\section{HENRY F. HELMHOLZ, M.D. ROCHESTER, MINN.}

The present study is concerned with an attempt to determine the direct mode of action of several of the more commonly used drugs in the treatment of pyelitis. The action of the alkalis, of hexamethylenamin and of phenyl salicylate has been studied.

First, it must be said that pyelitis, in most instances, is a self limited disease, which may disappear almost as rapidly as it appears. It is sometimes discovered accidentally by routine examination of the urine. At other times, it may be associated with very severe symptoms, being, even, rapidly fatal, owing to renal complications. Our inability to distinguish between pyelitis with and without renal involvement makes it extremely difficult to differentiate, at the onset, the severe from the mild; at present, the clinical course is the sole criterion. This difficulty practically rules out the possibility of comparing the various modes of treatment by studying a given number of cases. In treating the same patient first in one way and then in the other, it is impossible to determine whether the disease would not have cleared up if the first treatment had been continued instead of the second being instituted.

In taking up the value of alkalization from the historical standpoint, Thompson of Edinburgh seems to have first emphasized the value of alkalization of the urine on purely empiric grounds. He has given no theory of action, nor has any one given us a satisfactory explanation of its value. In a recent article on the treatment of pyelitis, Browning ${ }^{1}$ says, "The change in reaction of the urine causes the tissues to be bathed in a fluid which is more bland, and thereby enables them to combat the infection more effectually." Normal urine is slightly acid, so that the normal mucous membrane of the urinary tract is developed to withstand these degrees of acidity without damage. Whether this applies also to an inflamed mucous membrane has not been determined.

The question that naturally arises is whether the alkalization is merely a means of increasing urinary output and flushing the passages, or whether it has a definite specific effect. The greatest alkalinity that has been found in urine of human beings is $p_{\mathrm{H}} 8.6$, the same as a saturated solution of sodium bicarbonate. Since in this alkalinity the colon bacillus grows luxuriantly, it is evident that this degree of alkalinity is insufficient to influence the clinical condition by its action on the growth of this organism. The growth of the colon bacillus is not inhibited until the hydrogen ion concentration reaches from 9.2 to 9.6 . Since, at the acid end, inhibition of growth occurs at from $p_{\mathrm{H}} 4.6$ to 5 , and urines with hydrogen ion concentration as high as 5 may occur, the inhibitory action by acidity alone would be possible.

After the disappearance of the general symptoms, fever, malaise, anorexia, and so forth, the urinary findings in pyelitis are usually as marked as before; so that it seems possible that the alkalinity might prevent the formation and absorption of toxic products producing the general symptoms. Experiments were conducted

* From the Section on Pediatrics, Mayo Clinic.

* Read before the Section on Diseases of Children at the Seventy Third Annual Session of the American Medical Association,' St. Louis, May, 1922

1. Browning. C. H.: The Diagnosis and Treatment of Nonvenereal Pyogenic Infections of the Urinaty Tract. with Special Reference to Urinary Antiseptics, Glasgow M. J. 97:38.45 (Jan.) 1922. in order to determine whether there is a difference in toxicity when the colon bacillus is grown in acid broth and when in alkaline broth. Bacilli were grown at an acidity $p_{\mathrm{H}} 5.4$ and at an alkalinity of $p_{\mathrm{H}} 8.6$ for twentyfour hours; the cultures were then filtered, and the filtrate was injected intravenously into rabbits, similar amounts of the acid and of the alkaline broth being used as control. The reactions were practically identical. Both the alkaline and the acid broth produced a febrile reaction of from 2 to 5 degrees; the filtrates caused a slight rise of temperature and then a marked lowering of temperature. The reactions of alkaline and acid filtrates were practically identical. The difficulty of growing the organisms at a definite hydrogen ion concentration is that ordinary alkaline mediums in which there is sugar rapidly become acid, and acid mediums more acid. Sugar-free alkaline mediums become more alkaline and sugar-free acid mediums tend to become alkaline. In these experiments, an excess of sodium bicarbonate was added to the alkaline broth and a buffer mixture to the acid broth, so that the hydrogen ion concentration after twenty-four hours was within two tenths of that before inoculation.

A number of observers have said that if alkalization does not clear up the infection, the rapid change to the use of hexamethylenamin and acidification proves effective. This treatment may owe its effectiveness entirely to the action of the hexamethylenamin; on the other hand there is a possibility that the beneficial effect is due to the lag observed in the growth of bacteria when their culture medium is changed. The lag is the time required for the bacteria to acclimate themselves to their new surroundings. The change from acid to alkali, and vice versa, might tend to retard the development of the colon bacillus. We, therefore, studied the rapidity of multiplication of the colon bacillus when grown in acid broth and transplanted to alkaline, as compared with that of one that was grown in alkaline broth for numerous generations, and vice versa. No striking differences were observed in this series of experiments when they were carried on in mediums without a buffer. As the growth of the colon bacillis changed the hydrogen ion concentration of the medium very rapidly (so that if the concentration at the time of inoculation is 8.6, after a few hours of growth it may be only 6.4), it was thought best to repeat the experiments in mediums with buffer mixtures, so that the reaction, after twenty-four hours' growth, would be very nearly the same as before inoculation. It was not possible in any of the experiments to demonstrate any appreciable lag when the colon bacillus was transferred from an acid to an alkaline medium, and only a very slight lag when it was transferred from an alkaline to an acid. These experiments do not exclude any effect that the alkaline mode of treatment may have on the formation of bactericidal substances by the mucous membrane.

A special effort has been made to determine whether there was any action of hexamethylenamin at the level of the pelvis. It is well known that urine from the bladder, if acid, can be rendered sterile by hexamethylenamin. According to the experiment of Shohl and Deming, ${ }^{2}$ with a hydrogen ion concentration of 5.2 and a total urinary output of 1,500 c.c., a dosage of $3 \mathrm{gm}$. of hexamethylenamin will produce formaldehyd in two hours sufficient to kill 90 per cent. of all bacteria.

2. Shohl, A. T., and Deming, C. L.: Hexamethylenamin: Its Quantitative Factors in Therapy, J. Urology 4: 419 (Oct.) 1920. 
They have also shown that the nearer the reaction of the urine approached neutrality, the lower was the concentration of formaldehyd and antibactericidal action. With a $p_{\mathrm{H}} 6$, insufficient formaldehyd was formed even in twenty-four hours to kill all bacteria. At neutrality, there was no production of formaldehyd. In infants, the urine rarely remains in the bladder more than from one to one and one-half hours. Even if conditions of acidity in the urine of the bladder are ideal and the hexamethylenamin is sufficient, it would be almost impossible to obtain a bactericidal concentration of formaldehyd because of the lack of time.

I recently observed a child who had suffered from pyelitis for three years. Ureteral catheterization, some months before, showed bilateral infection of the renal pelvis. The urine, on first examination, contained innumerable bacilli, but only a few pus cells. The child was put to bed, fluid was forced, and 15 grains of hexamethylenamin and 20 grains of sodium acid phosphate were given every six hours. In spite of the sodium acid phosphate, the hydrogen ion concentration of the urine remained about 7 , varying from 6.8 to 7.4 . At the end of eight days, the urine of the bladder was sterile, but numerous bacteria could be seen in the centrifuged specimen. A few days later, the child underwent cystoscopy, and urine was obtained from both ureters and from the bladder, and cultures were taken. All the cultures were sterile, and the hydrogen ion concentration was 7.4. What had caused the disappearance of the infection? The urine was alkaline; the formaldehyd could never be in sufficient concentration to be bactericidal. There is, of course, a possibility that disappearance of infection was merely a concidence, or due to rest in bed and forcing of fluids, but the three year history is rather against such an assumption. It raises the question of a possible action of hexamethyl-. enamin in alkaline mediums. A particularly interesting experiment was made to determine the condition of the urine from the ureters, when the urine from the bladder contained sufficient formaldehyd to sterilize it ; and to determine the length of time required to render the urine from the ureters sterile. Thus far fourteen cases have been studied, but an appropriate, deciding case has not been found.

Experiments with the use of phenyl salicylate (salol) have been somewhat limited, because indications did not warrant further investigation. They consisted of two cases of pyelitis in which the dose of phenyl salicylate was increased gradually to a point at which it was unjustifiable to go farther.

In the case of one patient suffering from pyelitis, the dose of phenyl salicylate was increased to $5 \mathrm{gm}$. daily, without any effect on the bacteria in the urine. With this dose, the urine was not bactericidal ; and it turned rapidly dark on standing; indicating the presence of pyrocatechin and hydrochinon. In the case of another patient, the dose was increased gradually to $4 \mathrm{gm}$. daily, at which time there was rapid change of the color of the urine, to a dark brown. In neither instance was any antiseptic action observed. Since these doses are much higher than those usually advised for the treatment of pyelitis, I feel warranted in maintaining that, in the doses usually given, phenyl salicylate has practically no value in rendering the urine sterile by its direct action on bacterial growth.

From a review of the literature of pyelitis, particularly of its treatment in infancy, the impression is gained that few observers have found hexamethylenamin of much value. In most articles on the subject, alkalization is the treatment of choice in the acute cases. However, there is no evidence that alkalization has any direct specific effect, except, possibly, its diuretic action. The possible effect of alkalization on the tissues is not considered in this paper, it being the subject of a separate experimental study.

\section{SUMMARY}

In acute cases of pyelitis, the alkalis are useful, but we have no evidence of any direct specific action. Hexamethylenamin has a very definite bactericidal action in the bladder, but whether this applies also to the pelvis of the kidney has not been demonstrated. Phenyl salicylate does not have antiseptic properties in the doses given.

\section{UROLOGIC SURGERY IN PEDIATRICS *}

\author{
HERMAN L. KRETSCHMER, M.D. \\ Genito-Urinary Surgeon, Alexian Brothers Hospital; Urologist, Presby- \\ terian Hospital; Consultant in Urology, U. S. Veterans' \\ Hospital No. 76; Assistant Surgeon, Chil- \\ dren's Memorial Hospital \\ CHICAGO
}

It may be said without fear of contradiction that the urologic study of pediatric cases has not received the consideration that is due so important a subject. This is practically an undeveloped province of medicine, and, as evidence of this statement, attention may be called to the scant references, both in pediatric and in genitourinary literature. In this country, Beer, ${ }^{1}$ Hyman, ${ }^{2}$ Hinman, ${ }^{3}$ Stevens, ${ }^{4}$ Thomas and Tanner, ${ }^{,}$and Helmholz and $I^{\circ}$ have made a study of the subject-not a large showing. Hence, it appears to me that this province for clinical study has been decidedly neglected, though the problems and possibilities undoubtedly are many and of great variety. But to achieve gratifying results, the closest cooperation between the pediatrician, general practitioner and genito-urinary surgeon is necessary. This alliance effected, I am ready to admit, without being unduly enthusiastic, that pediatric urology will be the open sesame for extensive work for both pediatricians and urologists.

On reflection, it appears that there is no really sound reason for this backwardness of pediatric urology. A perusal of the table of diagnoses (Table 2) shows that the lesions of the genito-urinary tract in children differ in no way from the various pathologic conditions fornd in adults, except as regards those incident to age, such as carcinoma. Then what are the reasons for this failure to keep abreast of the advances made in urology in adults? The reasons are:

1. The general practitioners and pediatricians who attend infants and children have not availed themselves of the advantages and opportunities of urologic studies because of the difficulty in eliciting symptoms in these patients, and probably because they are not familiar with the possibilities inherent in this subject.

* Read before the Section on Diseases of Children at the SeventyThird Annual Session of the American Medical Association, St. Louis, May, 1922.

1. Beer: Am. J. Surg., March, 1911

2. Hyman, A.: Urinary Tract Surgery, Am. J. Dis. Child. 15: 116 (Feb.) 1918 .

3. Hinman, F.: Cystoscopic Study of Urologic Conditions in Children, Am. J. Dis. Child. 17: 305 (May) 1919 .

4. Stevens, W. E.: Diagnosis and Treatment in Pathologic Conditions of Urinary Tract in Children: Modern Methods, J. A. M. A. 77: 1081 (Oct. 1) 1921.

5. Thomas,
March) 1922 .

March) 1922. $H$. 6. Helmbolz, H. F., and Kretschmer, H. L.: Treatment of Pye
in Infancy and Childhood, J. A. M. A. $75: 1303$ (Nov. 13) 1920 . 